\title{
H-MR Spectroscopy of the Anterior Cingulated Cortex: Usefulness in the Prediction of Patients That Will Benefit from a Cognitive Behavioural Therapy in the Treatment of Chronic Pain
}

\author{
Sei Fukui ${ }^{*}$, Masahiro Yoshimura ${ }^{2}$,Katsunori Miyata ${ }^{2}$, Nishiyama Junji ${ }^{3}$ \\ ${ }^{1}$ Pain Management Clinic, Department of Anesthesiology, Shiga University of Medical Science Hospital, Otsu, Japan \\ ${ }^{2}$ Department of Anesthesiology, Shiga University of Medical Science Hospital, Otsu, Japan \\ ${ }^{3}$ Department of Psychosomatics, Shiga University of Medical Science Hospital, Otsu, Japan \\ Email: *sei@belle.shiga-med.ac.jp
}

Received February 1, 2013; revised February 21, 2013; accepted February 28, 2013

\begin{abstract}
Anterior cingulated cortex (ACC) is involved in "the state in which patients do not care much about pain despite its presence" which is a goal of psychosomatic treatment. To investigate the absolute concentration of N-acetylaspartate (NAA) in the anterior cingulated cortex (ACC) as predictors of patients that may benefit from cognitive behavioural therapy in the treatment of chronic pain. Proton magnetic resonance spectroscopy $\left({ }^{1} \mathrm{H}-\mathrm{MRS}\right)$ was performed with a 1.5 T MR system on a voxel in the bilateral ACC in 85 chronic pain patients and 20 age-matched normal control subjects. Eighteen out of $24(75.0 \%)$ patients whose NAA concentration decreased significantly in the ACC, respectively, compared to the mean NAA concentration of the normal control subjects, needed cognitive behavioural therapy. Our results suggest that decreased NAA concentration in the ACC is associated with the necessity of cognitive behavioural therapy. ${ }^{1} \mathrm{H}$-MRS may serve as a useful non-invasive tool for evaluating chronic pain patients.
\end{abstract}

Keywords: Magnetic Resonance Spectroscopy; Chronic Pain; Anterior Cingulated Cortex; N-Acetylaspartate

\section{Introduction}

In recent years, studies using brain function imaging have gradually clarified the brain regions associated with pain leading to a more objective evaluation of pain [1]. The anterior cingulated cortex (ACC) have been demonstrated to be involved in cognitive-evaluative and affective-motivational aspects of pain in several functional neuroimaging studies including those using functional magnetic resonance imaging (fMRI) [1,2]. Studies using voxel-based morphometry (VBM) or fMRI in patients with chronic pain have shown atrophy of the ACC [3], and activation of ACC have been produced by virtual pain stimulation using a video [4]. Thus, functional changes in the ACC are considered to be closely involved in chronic pain [1-4].

It was reported that patients no longer showed psychological suffering despite the presence of pain after ACC resection as treatment for chronic pain [5]. Other studies have also shown a marked increase in blood flow in the ACC and pain relief during electrical stimulation of the cerebral cortex [6], ACC activation by the anticipation

"Corresponding author. of pain [7], and activation of the ACC by placebo administration [8]. In patients with central pain, a functional decrease in the ACC is considered to be the cause of specific unpleasantness [9], and ACC activity was reported to be correlated with the degree of pain and unpleasantness [9]. A recent study using Proton magnetic resonance spectroscopy $\left({ }^{1} \mathrm{H}-\mathrm{MRS}\right)$ showed suffering in neuropathic pain patients after spinal cord injury are associated with the level of N-acetylaspartate (NAA) in the ACC [10]. These findings also suggest that ACC is involved in "the state in which patients do not care much about pain despite its presence" which is a goal of psychosomatic treatment.

We measured neural activity in Brodmann Area 24 (BA24) in the ACC, using ${ }^{1} \mathrm{H}-\mathrm{MR}$ spectroscopy $\left({ }^{1} \mathrm{H}-\right.$ MRS) to evaluate the effect of chronic pain. MRS is a noninvasive method for the measurement of metabolites in the brain using an MRI system. N-acethyl-aspartate (NAA) obtained using ${ }^{1} \mathrm{H}$-MRS is amino acid specific to and present only in neurons $[11,12]$. Furthermore, there is no age-related differences on the level of NAA in the ACC and PFC [11,12]. Therefore, it is clinically used as a noninvasive parameter of local neural function in the 
brain for the differential diagnosis of Alzheimer's disease [13] and post-traumatic stress disorder (PTSD) [14].

In this study, to investigate whether the absolute concentration of NAA in the ACC can be a valuable evaluation tool of chronic pain that may benefit from a cognitive behavioural therapy (CBT), we investigated the absolute concentration of NAA in the ACC bilaterally, of chronic pain patients and healthy comparison subjects by ${ }^{1} \mathrm{H}-\mathrm{MRS}$.

\section{Materials and Methods}

\subsection{Subjects}

The subjects consisted of 85 patients ( 41 males, 44 females, ages $17-83$ years, $55.91 \pm 15.02$ years) with chronic pain (neuropathic pain, 20; complex regional pain syndrome: CRPS, 20; chronic low back pain, 20; whiplash syndrome, 9; other chronic pain, 16) who were referred to the pain management clinic of the Department of Anesthesiology of the Shiga University of Medical Science Hospital. The left side was affected in 24 patients, and the right side was affected in 27 . The duration of pain was 4 - 396 months. Psychological approach, such as a cognitive behavioral approach by psychologist was determined by multidisciplinary conference with psychologist. These patients were compared with 20 age- and sex-matched healthy controls (11 males, 9 females, ages 17 - 79 years). They were screened with a questionnaire and an interview, and those with current or a history of mental disorder, neurological disease or head trauma were excluded.

This study was approved by the ethics committee of Shiga University of Medical Science Hospital and informed written consent for participation in this study was obtained from all patients and volunteers.

\subsection{MR Imaging}

${ }^{1} \mathrm{H}-\mathrm{MRS}$ examinations were performed at $1.5 \mathrm{~T}$ (SIGNA MR System, General Electric, Milwaukee, WI, USA) using a standard circular polarized head coil. The voxels of interest were selected in the bilateral ACC region on sagittal and coronal T1-weighted images of the head (Figure 1). ${ }^{\mathrm{I}} \mathrm{H}-\mathrm{MRS}$ spectra were obtained using the simulatedecho method (STEAM) with chemical shift selective saturation (CHESS) pulse sequence with a repetition time (TR) of $2 \mathrm{sec}$, echo time (TE) of $30 \mathrm{msec}$ and 96 acquisitions in the anterior cingulated cortex.
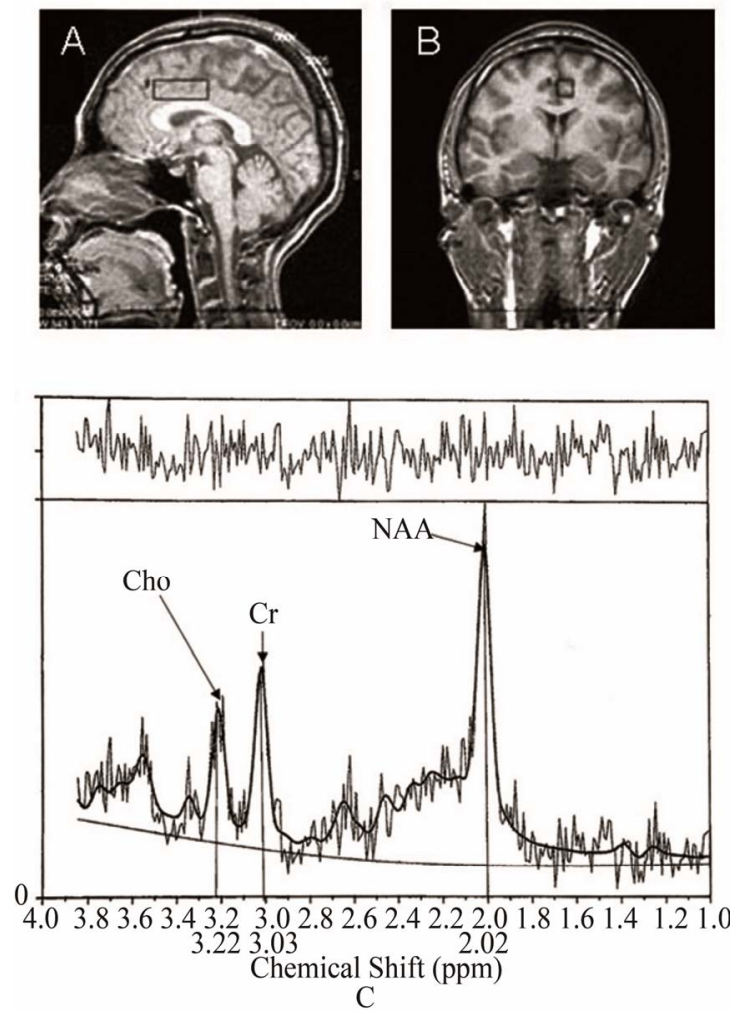

Figure 1. Axial and sagittal MRI scans showing the location of the spectroscopic voxel in the prefrontal cortex and anterior cingulate cortex. ${ }^{1} \mathrm{H}$-MRS spectra from the anterior cingulated cortex of a chronic pain patient. ROIs in the ACC were selected on T1-weighted images. MR spectra obtained in each region were analyzed using the LC model. NAA, N-acetyl-aspartate; $\mathrm{Cr}$, creatine and phosphocreatine; Cho, choline-containing compounds. Chemical shifts are indicated in parts per million (ppm). 
Based on the peaks of spectra, the NAA concentrations were determined.

Spectral analysis was performed using linear combination model (LC model) software [15], which yields concentrations of NAA (Figure 1). Brain voxels of interest were chosen in gray matter of the ACC (BA 23) from the axial plane by a skilled neuroradiologist.

\subsection{Evaluation Items and Statistical Analysis}

The intensity of pain in the patients was evaluated using a Visual Analogue Scale (VAS). VAS levels of pain severity were rated as $0-100 \mathrm{~mm}(0=$ no pain; $100 \mathrm{~mm}=$ maximal pain). The Hospital Anxiety Depression (HAD) test [16] was performed, and the severity of anxiety and depression associated with pain in the patients was evaluated.

In each region of interest, the NAA concentrations ipsilateral and contralateral to the affected limb were compared to determine whether there was a hemisphere predominance. The possible correlations between the NAA concentration in each region and the intensity of pain, duration of disease, or pain-associated affective-motivational changes were evaluated. We compared the absolute concentration of NAA in the ACC bilaterally, of chronic pain patients who needed CBT and those that did not require $\mathrm{CBT}$. The NAA concentrations were also compared between the patients and control group.

The NAA concentrations are expressed as the mean \pm standard deviation, and the two groups were statistically

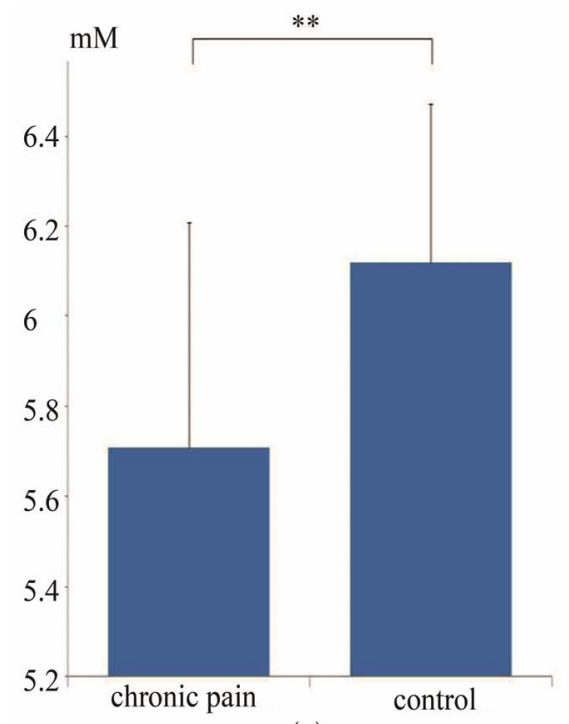

(a) compared using Mann-Whitney's test. $\mathrm{P}<0.05$ was regarded as significant.

\section{Results}

The affected side and NAA concentration in each region (hemispherical predominance):

In the left side-affected patients $(n=24)$, there was no significant difference in the NAA concentration between the left and right ACC (right ACC; $5.55 \pm 0.54$, left ACC; $5.49 \pm 0.42)$. In the right side-affected patients $(\mathrm{n}=27)$, there was no significant difference in the NAA concentration between the left and right ACC (right ACC; 5.56 \pm 0.44 , left ACC; $5.55 \pm 0.51)$. No significant difference $(\mathrm{P}>0.05)$ was observed between NAA concentrations ipsilateral to the affected side and those contralateral to the affected either in the ACC in chronic pain patients.

In the healthy control subjects $(\mathrm{N}=20)$, the mean left and right NAA concentration of the ACC was $6.12 \pm$ 0.35 , and the range was 5.77 to 6.47 . There was no association between age and the mean NAA concentration on the left and right sides in the ACC in the control group.

Compared with the 20 healthy controls, the NAA concentration was significantly (mean $\pm 2 \mathrm{SD}$ ) lower in the ACC in 22 of the 68 patients with chronic pain.

In the chronic pain patients $(\mathrm{N}=85)$, the mean NAA concentration $(5.70 \pm 0.50)$ of the ACC was significantly lower in the patients than in the control group (6.12 \pm 0.35) (Figure 2).

Twenty-four out of 85 chronic pain patients required a

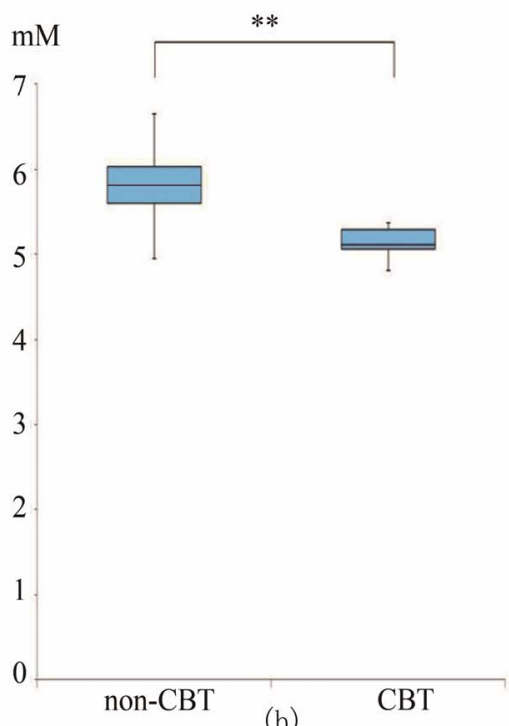

(b)

Figure 2. (a) Concentration of NAA in the ACC in chronic pain patients and control group; (b) The mean NAA concentration of the ACC in chronic pain patients who required a cognitive behavioural therapy performed by a psychologist and controls. In chronic pain patients $(\mathrm{N}=85)$ the mean NAA concentration $(5.70 \pm 0.50)$ in the ACC was significantly lower in the patient than in the control group $(6.12 \pm 0.35)(a)$. The mean NAA concentration $(5.16 \pm 0.24)$ of the ACC in chronic pain patients who required CBT performed by psychiatrist $(\mathrm{N}=24)$ was significantly lower than the NAA concentration $(5.88 \pm 0.44)$ in patients not needing CBT $(\mathrm{N}=61)$. 
cognitive behavioral approach performed by a psycologist. Eighteen of $24(75.0 \%)$ patients that had a decrease in NAA concentration in the ACC required a cognitive behavioral approach performed by psychologist.

The mean NAA concentration $(5.16 \pm 0.24)$ of the ACC of chronic pain patients who required CBT performed by a psychologist $(\mathrm{N}=24)$ was significantly lower than the NAA concentration $(5.88 \pm 0.44)$ in patients that did not need CBT $(\mathrm{N}=61)$ (Figure 2).

There was no correlation between the intensity of pain, the pain duration and the NAA concentration in ACC. There was no significant difference in the NAA concentration of the ACC between the patient group with marked anxiety $(\mathrm{n}=17$; anxiety $>11)(\mathrm{ACC} ; 5.70 \pm 0.43)$ in the HAD test and the patient group without anxiety $(\mathrm{n}=$ 68 ; anxiety < 10) (ACC; $5.79 \pm 0.48)$. There was no significant difference in the NAA concentration between the patient group showing marked depression associated with pain $(\mathrm{n}=27$; depression $>11)(\mathrm{ACC} ; 5.68 \pm 0.47)$ in the $\mathrm{HAD}$ test and the patient group without depression $(\mathrm{n}=$ 58 ; depression < 10) $(\mathrm{ACC} ; 5.66 \pm 0.48)$.

\section{Discussion}

${ }^{1} \mathrm{H}-\mathrm{MRS}$ is a noninvasive method for the measurement of biological metabolites that are difficult to morphologically evaluate $[11,13,17,18]$. NAA is localized in neurons at high concentrations and therefore is correlated with the density of normal neurons $[11,13,14]$. In ACC, the NAA concentrations ipsilateral to the affected limb were similar to those contralateral to the affected limb, showing no hemispherical predominance. Further, Coghill et al. [19] reported bilateral activation of ACC following painful stimulation. Therefore, in this study, irrespective of the affected side, the mean NAA concentration on the left and right sides of ACC was used for evaluation.

Reductions of NAA in the ACC were reported to be specifically linked to the presence of chronic pain with spinal cord injury [17]. A study using ${ }^{1} \mathrm{H}-\mathrm{MRS}$ in patients with diabetic neuropathy showed a decrease in the NAA concentration in the ACC in patients with marked pain [18]. Thus, the decrease in the NAA concentration suggests a decrease in the function of normal neurons in the ACC due to persistent unpleasantness associated with pain. In this study, the chronic pain patient group showed a significant decrease in the NAA concentration in ACC, compared to normal controls. Evaluation of ACC function may be a key to the successful treatment of chronic pain.

In the clinical setting, we often encounter patients with chronic pain showing depression and anxiety in which these affective changes further modify pain. However, the results of this study showed no significant difference in NAA concentration in the ACC in the patient group with marked anxiety and depression associated pain. There was no association between the intensity of pain, duration of pain, depression, anxiety and NAA concentration in ACC.

In this study, cognitive behavioural therapies were necessary in patients showing a decrease in the NAA concentration in the ACC. Our results using ${ }^{1} \mathrm{H}$-MRS suggest that the decreased NAA concentration of the ACC may be related to the necessity for CBT.

After treatment using a CBT performed by psychologist, although the intensity of pain remained the same, "pain obsession" decreased which led to psychological relief. When patients do not care as much about the pain, management for chronic pain may be more successful.

Our results using ${ }^{1} \mathrm{H}-\mathrm{MRS}$ suggest that the chronic pain patients who need CBT tend to have decreased neural activity in the ACC. Our results suggest that ${ }^{1} \mathrm{H}-\mathrm{MRS}$ is a noninvasive method that does not burden patients and is also useful for selecting treatment methods, and can be a new evaluation method in patients with chronic pain.

\section{Acknowledgements}

This work was funded by a Grant-in-Aid for Scientific Research (C).

\section{REFERENCES}

[1] I. Tracey, "Imaging Pain," British Journal of Anaesthesia, Vol. 101, No. 1, 2008, pp. 32-39. doi:10.1093/bja/aen102

[2] A. V. Apkarian, M. C. Bushnell, R. D. Treede and J. K. Zubieta, "Human Brain Mechanism of Pain Perception and Regulation in Health and Disease," European Journal of Pain, Vol. 9, No. 4, 2005, pp. 463-484. doi:10.1016/j.ejpain.2004.11.001

[3] A. V. Apkarian, Y. Sosa, S. Sonty, R. M. Levy, R. N. Harden and D. R. Gitelman, "Chronic Back Pain Is Associated with Decreased Prefrontal and Thalamic Gray Matter Density," Journal of Neuroscience, Vol. 24, No. 46, 2004, pp. 10410-10415. doi:10.1523/JNEUROSCI.2541-04.2004

[4] T. Ushida, T. lkemoto, S. Taniguchi, K. Ishida, Y. Murata, W. Ueda, S. Tanaka, A. Ushida and T. Tani, "Virtual Pain Stimulation of Allodynia Patients Activates Cortical Representation of Pain and Emotions: A Functional MRI Study," Brain Topography, Vol. 18, No. 1, 2005, pp. 27 35. doi:10.1007/s10548-005-7898-8

[5] K. D. Davis, E. Taub, F. Duffner, A. M. Lozano, R. R. Tasker, S. Houle and J. O. Dostrovsky, "Activation of the Anterior Cingulated Cortex by Thalamic Stimulation Patients with Chronic Pain: A Positron Emission Tomography Study," Journal of Neurosurgery, Vol. 92, No. 1, 2000, pp. 64-69. doi:10.3171/jns.2000.92.1.0064

[6] R. Peyron, I. Faillenot, P. Mertens, B. Laurent and L. Garcia-Larrea, "Motor Cortex Stimulation in Neuropthic Pain. Correlations between Analgesic Effect and Hemo- 
dynamic Changes in the Brain: A PET Study," Neuroimage, Vol. 34, 2007, pp. 310-331.

doi:10.1016/j.neuroimage.2006.08.037

[7] T. Koyama, J. G. McHaffie, P. J. Laurienti and R. C. Coghill, "The Subjective Experience of Pain: Where Expectations Become Reality," Proceedings of the National Academy of Sciences of the United States, Vol. 102, 2005, pp. 12950-12955. doi: 10.1073/pnas.0408576102

[8] T. D. Wager, J. K. Rilling, E. E. Smith, A. Sokolik, K. L. Casey, R. J. Davidson, S. M. Kosslyn, R. M. Rose and J. D. Cohen, "Placebo-Induced Changes in FMRI in the Anticipation and Experience of Pain," Science, Vol. 303, 2004, pp. 1152-1167. doi:10.1126/science.1093065

[9] R. Peyron, L. Garcia-Larrea, M. C. Grégoire, P. Convers, F. Lavenne, L. Veyre, J. C. Froment, F. Mauguiere, D. Michel and B. Laurent, "Allodynia after Lateral-Medullary after Lateral-Medullary (Wallenberg) Infract: A PET Study," Brain, Vol. 121, No. 2, 1998, pp. 345-356. doi:10.1093/brain/121.2.345

[10] E. Widerström-Noga, P. M. Pattany, Y. Cruz-Almeida, E. R. Felix, S. Perez, D. D. Cardenas and A. Martinez-Arizala, "Metabolite Concentrations in the Anterior Cingulated Cortex Predict High Neuropathic Pain Impact after Spinal Cord Injury," Pain, Vol. 154, No. 2, 2013, pp. 204-212. doi:10.1016/j.pain.2012.07.022

[11] I. D. Grachev and A. V. Apkarian, "Aging Alters Regional Multichemical Profile of the Human Brain: An in Vivo 1H-MRS Study of Young versus Middle-Aged Subjects," Journal of Neurochemistry, Vol. 76, No. 2, 2001, pp. 582-593. doi:10.1046/j.1471-4159.2001.00026.x

[12] H. Reyngoudt, T. Claeysa, L. Vlericka, S. Verledenc, M. Acoua, K. Deblaerea, Y. D. Deened, K. Audenaert, I. Goethals and E. Achten, "Age-Related Differences in Metabolites in the Posterior Cingulate Cortex and Hippocampus of Normal Ageing Brain: A 1H-MRS Study," European Journal of Radiology, Vol. 81, No. 3, 2012, pp. e223-e231. doi:10.1016/j.ejrad.2011.01.106
[13] F. Jessen, W. Block, F. Träber, E. Keller, S. Flacke, A. Papassotiropoulos, R. Lamerichs, R. Heun and H. H. Schild, "Proton MR Spectroscopy Detects a Relative Decrease of Nacetylaspartate in the Medial Temporal Lobe of Patients with AD," Neurology, Vol. 55, No. 5, 2000, pp. 684-688. doi:10.1212/WNL.55.5.684

[14] K. Mahmutyazicioglu, K. N. Konu, H. Ozdemir, N. Atasoy, L. Atik and S. Gündoğdu, "Evaluation of the Hippocampus and the Anterior Cingulated Gyrus by Proton MR Spectroscopy in Patients with Post-Traumatic Stress Disorder," Neuroradiology, Vol. 11, 2005, pp. 125-129.

[15] G. Helms, "Analysis of 1.5 Tesla Proton MR Spectra of Human Brain Using LC Model and an Imported Basis Set," Magnetic Resonance Imaging, Vol. 17, No. 8, 1999, pp. 1211-1218. doi:10.1016/S0730-725X(99)00058-2

[16] I. Bjelland, A. A. Dahl, T. T. Haug and D. Neckelmann, "The Validity of the Hospital Anxiety and Depression Scale: An Updated Literature Review," Journal of Psychosomatic Research, Vol. 52, 2002, pp. 69-77. doi:10.1016/S0022-3999(01)00296-3

[17] P. Stanwell, P. Siddall, N. Keshava, D. Cocuzzo, S. Ramadan, A. Lin, D. Herbert, A. Craig, Y. Tran, J. Middleton, S. Gautam, M. Cousins and C. Mountford, "Neuro Magnetic Resonance Spectroscopy Using Wavelet Decomposition and Statistical Testing Identifies Biochemical Changes in People with Spinal Cord Injury and Pain,' NeuroImage, Vol. 53, No. 2, 2010, pp. 544-552. doi:10.1016/j.neuroimage.2010.06.051

[18] L. Sqrensen, P. J. Siddall, M. I. Trenell and D. K. Yue, "Differences in Metabolites in Pain-Processing Brain Regions in Patients with Diabetes and Painful Neuropathy," Diabetes Care, Vol. 31, No. 5, 2008, pp. 980-981. doi: $10.2337 / \mathrm{dc} 07-2088$

[19] R. C. Coghill, C. N. Sang, J. M. Maisog and M. J. Iadarola, "Pain Intensity Processing within the Human Brain: A Bilateral, Distributed Mechanism," Journal of Neurophysiology, Vol. 82, 1999, pp. 1934-1943. 